\title{
Disponer las prácticas \\ Consumo, crédito y ahorro en un asentamiento del Gran Buenos Aires*
}

\author{
Arranging Practice \\ Consumption, credit and saving on a settlement in the \\ Metropolitan Area of Buenos Aires
}

Pablo Figueiro**

\begin{abstract}
Resumen: El presente artículo se propone indagar sobre las especificidades que adopta el uso del dinero en sectores subalternos a partir de un trabajo de campo realizado en un asentamiento de la localidad de José León Suárez, provincia de Buenos Aires. Se sostiene que en el cruce entre, por un lado, las modalidades que asume la percepción del ingreso en dicha población y, por el otro, los mecanismos formales e informales que regulan la relación con el dinero, se generan prácticas específicas de consumo, crédito y ahorro que tienden a profundizar las desigualdades existentes en dichos sectores. En este sentido, el trabajo plantea que las prácticas de consumo son estructuradas en torno a las condiciones de percepción del ingreso, es decir, a partir del trabajo del que se disponga - formal o informal -, la regularidad del pago - fijo o no y, en el primer caso, con que frecuencia temporal se percibe - y la forma del mismo - si se halla bancarizado o no - . Vinculado a esto, se desprenden distintas posibilidades de acceso a créditos en bancos, en casas de venta de bienes para el hogar, de "confianza" entre parientes y vecinos y/o en comercios del lugar- que suponen otras tantas formas de relación con el tiempo y de la vivencia y calculabilidad del mismo. Estas cuestiones, entrecruzadas por reglamentaciones, exclusiones y controles que operan modelando las formas que adopta el gasto, encuadran formas diferenciales del manejo del dinero y del ahorro.
\end{abstract}

Palabras claves: Prácticas económicas; Consumo; Gubernamentalidad; Sectores subalternos

\footnotetext{
*Este artículo es una versión revisada y modificada de la ponencia presentada en la VIII Reunión de Antropología del Mercosur, bajo el título Consumo, crédito y ahorro en un asentamiento del conurbano bonaerense: continuidades y especificidades sociales.

**Becario del Consejo Nacional de Investigaciones Científicas y Técnicas (Conicet), Argentina. Centro de Estudios Sociales de la Economía (Cese) del Instituto de Altos Estudios Sociales Universidad Nacional de San Martín. <pablofigueiro@gmail.com>.
}

\begin{tabular}{|l|l|l|l|l|l|}
\hline Civitas & Porto Alegre & v. 10 & n. 3 & p. 410-429 & set.-dez. 2010 \\
\hline
\end{tabular}




\begin{abstract}
The following paper is an attempt to explore some dimensions involved in the use of money by subaltern sectors from José León Suárez, province of Buenos Aires. We claim that the specific practices of consumption, credit and saving, that take place at the intersection between the perceptions about income and the formal and informal mechanisms regulating the relationship to money, tend to widen the inequalities found in those social sectors. In this sense, our paper claims that consumption practices are structured by perceptions around income, that is, the nature of the job involved whether formal or informal - the regularity and periodicity of wages, and the modality of wage payment - bank account or otherwise. Within this framework, we may find differential access to credit - in banks and retail stores as well as "credit rating" and "trust" among relatives and neighbors and small local stores - that entail associated ways of dealing with time both as an experience and something that can be calculated upon. All these dimensions, crisscrossed by regulations, exclusions and controls that shape the ways of spending, frame differential approaches to the use of money and saving.
\end{abstract}

Keywords: Economic practices; Governmentality; Consumption; Subaltern sectors

En la Comunicación "A” 2590 del 19 de septiembre de 1997, el Banco Central de la República Argentina (BCRA) estableció las normas que regirían la operatividad de las cuentas especiales para la remuneración de sueldos. Allí se dispuso que las entidades habilitadas que posean cajeros automáticos deberán abrir dichas cuentas a solicitud de los empleadores y a nombre de cada trabajador. Sólo se admitirá la acreditación de "las remuneraciones normales y habituales y otros conceptos derivados de la relación laboral" (Punto 4.4.3.). En cuanto a la extracción de fondos, el Banco Central autorizó el pago en supermercados y otros comercios adheridos mediante la tarjeta de débito, la cual debía proveerse sin cargo a cada titular de una cuenta. Asimismo, se permitió el pago de impuestos, servicios y "otros conceptos, a su vencimiento por cajero automático de la entidad o mediante el sistema de débito automático, sin límite de adhesiones". Cuando los empleadores y trabajadores acordasen la utilización de cuentas y/o servicios distintos a los previstos por la reglamentación, no regiría el carácter gratuito del servicio de acreditación. A partir de esto se comprende que las entidades realicen todo tipo de ofrecimientos - como seguros contra robos - por los que se cobran porcentajes sobre el total depositado.

Este cambio en la modalidad de efectuar las remuneraciones puede pensarse en términos económico - políticos antes que técnicos si tomamos en cuenta los efectos de poder que ejerce sobre la población. En efecto, mediante esta nueva modalidad para el pago de sueldos, automáticamente ingresaron al sistema bancario una ingente masa de asalariados - la totalidad de los empleados 
en relación de dependencia del país -, convirtiéndose en una clientela "cautiva" de los bancos y potenciales compradores de los demás productos y servicios que los mismos ofrecen, tales como tarjetas de crédito y préstamos personales. Por otra parte, los trabajadores han quedado inmersos en una red de información de la que dispone el sistema financiero, a través de la cual se clasifica la "calidad" de los deudores y se facilita o dificulta la obtención de créditos y de otros servicios, lo cual va de la mano con otros sistemas públicos - como la Central de Información que posee el BCRA en su página web - y privados tales como "Veraz"1 - En este sentido, puede rastrearse uno de los dispositivos a través de los cuales se gobiernan las prácticas monetarias de la población, puesto que la bancarización del salario supone un complejo entramado de disposiciones, accesibilidades, regularidades y controles que en su conjunto permiten una modalidad de consumo "electrónica" y mayoritariamente a crédito, lo cual, en su extremo, conduce a un endeudamiento permanente o "crónico" al reorganizar el tiempo en función de la posibilidad de desplazar a futuro el pago de artículos o servicios a los que puede accederse hoy.

\section{Cuadro 1: Evolución de las cuentas sueldo en millones de unidades (2000-2009)}

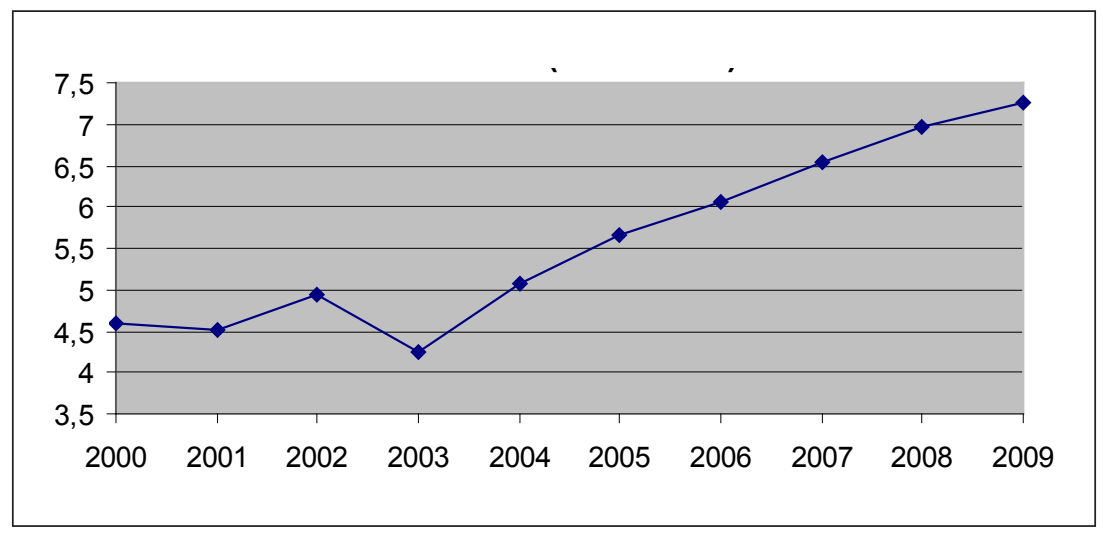

Fuente: Elaboración propia en base a datos del Banco Central de la República Argentina.

1 El "Veraz" es un servicio privado de información que otorga a quienes lo consultan un informe sobre el comportamiento de pago de personas y empresas, especificando todo lo relacionado con el incumplimiento de las obligaciones pasadas, tal como la emisión de cheques rechazados, servicios impagos, deudas fiscales, querellas judiciales relacionadas, etc. De esta forma, se ha transformado en una nómina temida por sus implicancias negativas a la hora de pedir financiamiento o de adquirir un servicio de abono periódico. 
Si presentamos este caso específico es porque ilustra cómo las prácticas económicas pueden ser objeto de una construcción y normalización y, por esto mismo, de un proceso rastreable. Pensar las prácticas requiere pensar la construcción de las mismas, el proceso por el cual llegaron a gozar de cierta naturalidad o, en otros términos, a ser invisibilizadas en lo que de contingentes tienen. El empleo del dinero no escapa a esta suerte, en tanto no se halla regido por una "racionalidad" ecuménica que guíe positivamente los usos sociales del mismo, lo cual es otra forma de decir que la racionalidad debe ser pensada en su construcción y que habrá tantas racionalidades como usos sociales haya, por caso, del dinero. ${ }^{2}$ Lo que nos interesa mostrar es cómo, de manera sutil, en las prácticas de intercambio y consumo pueden observarse los efectos concretos de una normalización a través de dispositivos que intentan gobernar los actos económicos en su capilaridad. Si la circulación de personas y de bienes no puede pensarse por fuera de los marcos institucionales, las convenciones culturales y las relaciones de poder (Reygadas, 2008), los efectos evidentemente desiguales que tendrán sobre las personas deberán verse en los casos concretos, es decir, en la cotidianidad de las prácticas, en la interrelación entre dichos mecanismos y las estrategias y signficaciones que los agentes adopten frente a los mismos, en función de los capitales específicos con los que cuenten. En este cruce, creemos, se inscriben las lógicas que subyacen a las economías domésticas.

En una investigación previa (Figueiro, 2009) nos hemos enfocado en las modalidades de consumo dentro de un asentamiento (el "22 de agosto") del Partido de General San Martín, provincia de Buenos Aires, donde, de una manera general, intentamos dar cuenta de las lógicas heterogéneas que dichas modalidades expresan. A partir de un trabajo de campo -que incluyó entrevistas etnográficas y observaciones participantes y no participantes- realizado entre los años 2007 y 2008, pudimos observar allí cómo la utilización del dinero se halla condicionada por una serie de mecanismos que en su conjunto tienden a orientar las prácticas económicas de la población. Si bien los fines últimos a los que estén dirigidos los gastos pueden obedecer a las más variadas motivaciones y lógicas (Zelizer, 2005b; Miller, 2000), las modalidades que asumen están regidas de tal manera entre los sectores con bajos recursos que

2 El análisis de Viviana Zelizer en relación al dinero (1997) muestra cómo las prácticas de diferenciación y de "marcación" de las monedas a partir de sus orígenes y de sus usos cotidianos contribuyen a crear "monedas múltiples", en las que se ponen en juego vínculos sociales que van más allá de la caracterización neutral del dinero ofrecida desde las perspectivas económicas clásicas. Para una revisión bibliográfica sobre los análisis del dinero, ver Dufy y Weber, 2009. 
tienden a profundizar la desigualdad existente con otros sectores sociales. En este sentido, las formas de percepción del ingreso y los andamiajes que regulan el acceso al crédito y al ahorro, son claves para comprender cómo se construye determinada relación con el dinero y con su utilización ${ }^{3}$.

A partir de los gastos que hemos considerado extraordinarios, es decir, aquellos cuyo fin no se halla en la reproducción cotidiana - y en contraposición a la extendida prenoción de que los pobres no pueden acceder a ciertos bienes por no contar con excedentes monetarios -, pudimos observar cómo los altos niveles de informalidad laboral y los magros ingresos que en la mayoría de los casos podían observarse, conducen a la utilización de mecanismos informales de financiamiento como única alternativa para el acceso a bienes que de otra manera les estarían vedados. Sin embargo, esto no debe abordarse presuponiendo la existencia de una correlación lógica previa, matematizable, entre las condiciones concretas de un individuo (como el nivel de ingresos o la posesión de bienes que puedan oficiar como garantías) y los condicionamientos o acreditaciones que se le exigen para acceder a determinado tipo de financiamiento en función del riesgo de incobrabilidad que supondría dicho individuo. Por el contrario, este cálculo, la forma de efectuarlo, supone una determinada relación de fuerzas que se cristaliza en una configuración específica de dispositivos tendientes a gobernar las economías domésticas en un sentido dado. Dicho de otra manera, el estímulo al consumo o al ahorro no es únicamente una cuestión matemática entre los ingresos, el riesgo y las tasas de interés, sino de los mecanismos a partir de los cuales se construye cierta naturalidad de las prácticas económicas.

El presente artículo se propone mostrar algunas de nuestras conclusiones en la investigación señalada, enfocándonos especialmente en los efectos que conllevan para las economías domésticas los mecanismos de percepción del ingreso y en cómo las condiciones de informalidad y precariedad laboral tienden a profundizar las desigualdades por la vía de los dispositivos que regulan el crédito y el ahorro.

\section{El campo: ingresos, crédito y relación con el futuro}

El asentamiento "22 de Agosto"4 surgió a partir de una ocupación de tierras - un basural ilegal a cielo abierto - en el año 1998, y se ubica en

\footnotetext{
3 A este respecto, puede leerse un pasaje de Foucault en el que analiza los dispositivos sutiles que se van creando a lo largo del siglo XIX en Francia para "controlar la economía de los obreros", de modo que "no sólo el tiempo de su vida laboral, sino el de su vida entera, podrá efectivamente ser utilizado de la mejor manera posible por el aparato de producción" (Foucault 2007c, p. 139).

4 Todos los nombres fueron modificados para preservar la privacidad de los vecinos del barrio y la confianza otorgada.
} 
las proximidades del relleno sanitario Zona Norte III de la Coordinación Ecológica del Área Metropolitana (CEAMSE), en el partido de San Martín de la provincia de Buenos Aires. El nivel de urbanización es acotado, aunque en ascenso, no contando con servicios legales ni asfalto. El tendido de luz, agua ${ }^{5}$ y televisión por cable fue realizado por los mismos vecinos o por personas que trabajan en las empresas prestadoras de dichos servicios, en ambos casos de manera ilegal. Existen múltiples construcciones de material que se fueron incrementando en el transcurso de los años, entre las cuales unas pocas llegan a alcanzar los dos pisos. No obstante, estas casas conviven con "ranchos" de chapa y madera, presentando cierta heterogeneidad edilicia, aunque tomado en su conjunto el barrio no exhibe cambios dramáticos en su morfología. La extensión del asentamiento se halla en continua expansión debido a los rellenos que fueron realizándose en las zonas más bajas, contando hasta el momento de la investigación con unas 16 manzanas aproximadamente. Si bien no existen registros censales, los entrevistados refieren que la mayoría de los vecinos realiza "changas" o incursiones en la "quema" del CEAMSE a fin de recolectar objetos (desde electrónicos desechados por las propias empresas hasta comida envasada y materiales de bronce) que puedan utilizarse para el consumo directo o bien para su venta a terceros. En general, estas referencias coinciden con las labores mayoritariamente informales y precarizadas que pudimos observar durante el trabajo de campo.

Sin extendernos en los innumerables detalles y pormenores que necesitaríamos para una descripción de la vida en el barrio, para la presente bastará con indicar que conjuntamente con los niveles de precarización laboral señalados y los escasos ingresos que logran obtener, conviven una serie de gastos que no pueden ser explicados sin un entramado de relaciones y de mecanismos que hagan posible el acceso a algún tipo de financiamiento. Por un lado nos hallamos con circuitos comerciales ${ }^{6}$ que se extienden al interior del barrio y que combinan lógicas mercantiles con relaciones de confianza basadas mayoritariamente en los vínculos de vecindad, lo que da lugar a prácticas

5 En el caso del agua, las cañerías no se extienden hacia todas las casas, sino que existen canillas en determinados puntos a los cuales concurren los vecinos para acceder a la misma.

6 El concepto de circuitos comerciales, en el sentido otorgado por Viviana Zelizer (2005a), ofrece una alternativa a los enfoques que dicotomizan entre relaciones estrictamente mercantiles por un lado, y las personales por el otro. Por el contrario, los circuitos comerciales pueden entenderse como estructuras-puente que facilitan la coexistencia de ambas formas de vinculación social, es decir, de los lazos "impersonales" atribuidos a las operaciones comerciales, y de los "íntimos" propios de las relaciones afectivas. Si bien no todos los elementos que definen a un circuito comercial, en el sentido atribuido por Zelizer, se hallan presentes dentro del barrio, este concepto nos permite dar cuenta de la imbricación existente entre relaciones de cercanía y de confianza con aquellas otras de tipo meramente impersonal, definidas por el cálculo racional, el instrumentalismo y la búsqueda de interés. 
de fiado y regateo. En este sentido pueden entenderse ciertas compras en los comercios instalados en el interior del barrio y las efectuadas en la vía pública, donde es posible observar cierta flexibilidad en el pago al contado y en el precio $^{7}$. Sin embargo, esto es sólo una parte del consumo, puesto que también existen gastos que se efectúan en comercios ubicados fuera del asentamiento, en los que tiende a quedar circunscrito a la lógica impersonal mercantil, en el sentido de que no existe espacio para el regateo y el crédito se da sólo a condición de acreditar determinadas garantías de fiabilidad. En dichos casos es donde pueden observarse claramente cómo actúan los mecanismos a los que nos referimos, puesto que la situación de escasez que vive nuestro sector en cuestión no equivale a una imposibilidad de acceder a determinados bienes, sino más bien a una diferenciación en las condiciones bajo las cuales dichos bienes son adquiridos.

En la casa de Norma son trece las personas que viven: ella, su marido, los hijos de este y una nieta. Puede hacer una comida por día. Ella cobra $\$ 250$ mensuales por un plan social como contraparte del servicio que presta en el centro comunitario, en el que trabaja diariamente, en tanto que su marido es repositor de bebidas en una empresa distribuidora, donde gana unos 30 pesos diarios.

Después de conversar con Fernando, me puse a charlar con Norma, que estaba al lado nuestro y acotaba de vez en cuando. Es una mujer de carácter lacónico, más bien reacio, de semblante aguerrido, y siempre me deslumbra con algún comentario de lo más lúcido, como si acertara en el clavo cada vez que habla ${ }^{8}$. Estaba afligida porque a su marido se le habían roto dos "paletas" de gaseosa y cerveza en un reparto, y se lo iban a descontar todo, por lo que esa semana no iba a cobrar. "No sé cómo vamos a hacer, vamos a tener que resistir", concluyó. Luego me dijo que quería mejorar la casa, que tiene tres cuartos y una cocina grande, pero en la que todos juntos no pueden comer. Ella se preocupa por la nena más chica, que es la nieta de su marido. Los más grandes entienden cuando no hay comida y pueden arreglarse solos, pero "a la más chica ¿cómo le explico que no hay? (Nota de campo del 7/06/08).

\footnotetext{
7 Los vecinos nos han relatado cierta reticencia a comprar en los negocios del interior de barrio debido a los precios más elevados que manejan. Por otra parte, si bien existe el fiado en los mismos, el pago posterior se realiza sobre la base del precio de los bienes al día de cancelación, lo cual conlleva un beneficio adicional para el comerciante y es objeto de reproche por parte de los vecinos, quienes marcan moralmente este hecho en función de que los comerciantes son al mismo tiempo vecinos que se "enriquecen con el sudor" del resto. No obstante, es verdad que el pago a crédito existe y es posible, al margen de la percepción que se tenga del mismo.

8 Para evitar malos entendidos, diré que mi sorpresa ante estos comentarios no se debe a que presuponga una imposibilidad de los sectores subalternos a realizar apreciaciones valiosas, sino antes bien al hecho de que una persona reservada - mas no tímida - devele de repente un conjunto de cuestiones diversas y personales en una sola frase ("total”, parafraseando a Mauss) dicha en forma de sentencia, capacidad que muy poca gente posee.
} 
Esta escena es común entre los vecinos. De hecho, la familia de Norma cuenta con una entrada de dinero fija, casi una suerte para quienes viven de "changas". Sin embargo, un momento después empezamos a hablar de los celulares:

Tengo que arreglar el mío (el celular), pero fui a averiguar y sale $\$ 200$ (...). Este teléfono salía seiscientos y pico. Lo saqué en seis cuotas, pero con los intereses lo terminé pagando más de $\$ 700$.

Para comprender estas prácticas aparentemente contradictorias sin tratarlas como "patológicas" - como suele hacerse desde el sentido común-, debe tenerse en cuenta, en primer lugar, el marco en el que se llevan a cabo y que las estructuran. Vale decir que no se hallan en el vacío, sino que, como intentamos mostrar, se enmarcan en un sistema salarial y crediticio que organiza el manejo del dinero y del tiempo, y en este sentido, suponen formas diferenciales de cálculo y de relación con el futuro. Por otra parte, las condiciones sociales en el que se desarrolla la vida implican visiones del porvenir que no se corresponden con lo que la teoría económica dominante ha construido en torno al sujeto optimizador, puesto que la visión del futuro se halla siempre en relación a cuál sea el presente inmediato y a la percepción que tenga el agente de su lugar dentro de dicho presente.

En este sentido, la percepción que se tiene del futuro es clave para entender ciertas prácticas que no se hallan sujetas a la contabilidad eudaimónica de los placeres esperados y las penas presentes. Si las expectativas de concretar una satisfacción futura no tienen lugar dentro del cálculo, porque quizás ni siquiera entren en el campo de la posibilidad, el presente se vuelve el lugar de las satisfacciones. En estas condiciones, la periodicidad del ingreso se convierte en una forma de relacionarse con el tiempo, puesto que impone márgenes a partir de los cuales se demarcan las actividades y se delimitan las posibilidades e imposibilidades a las que se hallan sujetos.

En efecto, el trabajo que se tenga y la forma de pago son en gran medida el parámetro a partir del cual se establecen las contabilidades y las formas de cálculo que se llevan a cabo, puesto que organizan el tiempo presente y futuro en virtud de cuáles sean los gastos que deben asumirse en ese lapso y de cuánto se dispone para ello. No es lo mismo cobrar diariamente $\$ 30$ que $\$ 900$ de manera mensual, ya que la previsión tiene un horizonte menor y la expectativa de que algún imprevisto pueda suceder es más acotada en el primer caso. De esta forma, el pago semanal organiza de determinada manera las operaciones mentales en torno al futuro y a la utilización del dinero, en tanto que el pago quincenal o mensual de otra y la ausencia de trabajo, el "rebusque" o la "changa" de otra. Según observamos en el cambio de una situación a otra, 
cuanto mayor es el período que transcurre entre cobro y cobro, los recaudos para con el dinero son mayores y se requiere de un mayor esfuerzo por adecuar las prácticas a una contabilidad que hasta entonces era distinta.

Adrián: Yo me gastaba 500 "mangos" por quincena, entonces sabía que la plata me tenía que durar quince días. No me la podía gastar, porque yo estaba acostumbrado a que laburaba por mi cuenta con mi viejo y cada fin de semana tenía mi plata. Todos los fines de semana tenía plata. Y cuando empecé a laburar por quincena me costó un montón...

Pablo: ¿No gastártela en la primera semana?

A: Claro, y yo tenía ciento cincuenta para el pasaje seguro. Y bueno, ciento cincuenta ya no los tengo más. Esto no lo cuento. A ver, ¿cuánto me queda? Me queda esto. Necesito comer... ¿qué hace falta en la casa? Bueno, son doscientos menos. Y después lo que quedaba, cien, ciento cincuenta, doscientos, y bueno... ¿qué quiero hacer hoy? ¿Quiero salir con los pibes? Bueno, salgo con los pibes: unas "birras", unas pre-pizzas, unos pools... listo, joya. Y después si quedaba algo, bueno, la semana que viene saldré de vuelta.

Si tenemos en cuenta la enumeración en términos de prioridades que se realizan en esta entrevista, veremos que se encuentra primero el gasto en viáticos para poder seguir trabajando, luego la comida o ayuda en el hogar, y finalmente la "salida". El ahorro, en tanto atesoramiento, se halla ausente de la consideración, aunque no se descarta la posibilidad de que quede un excedente que pueda ser utilizado la semana siguiente, pero aún así queda dentro de un horizonte temporal acotado a lo inmediatamente asequible y no como un atesoramiento previsional o tendiente hacia algún fin posterior que esté por fuera de los señalados por el entrevistado. De esta manera, las condiciones que modelan el uso del dinero no se remiten sólo a la periodicidad del pago, sino también a otros andamiajes que regulan la utilización del mismo. En este sentido, la posibilidad de acceso a una Caja de Ahorro es referida como de importancia para el manejo del efectivo, puesto que estructuraría las prácticas de su utilización de acuerdo a la mayor o menor materialidad, cercanía y contacto con el mismo. La misma persona nos relató de la siguiente manera su vivencia respecto al ahorro:

Por ejemplo, yo, si tengo la plata en la mano, me pica. Yo cuando estaba trabajando, tenía una cuenta de ahorro. Yo la plata la tengo ahí. Yo si no la tengo en la mano, la tengo la plata. Ahora, cuando ya la saqué... bueno, ‘¿qué te hace falta?’ (Adrián, 22 de Agosto).

\footnotetext{
9 "Mango" es una expresión del lunfardo rioplatense para designar al dinero, en este caso, a los pesos argentinos.
} 
Tener la plata en el bolsillo se vivencia de una manera distinta a tenerla en el banco, para el caso de quienes han tenido alguna experiencia bancaria. El manejo que de ella se hace, su cálculo y su disposición temporal implican diferenciaciones en uno y otro caso, generando distintos grados de liquidez. Esto indicaría que el régimen salarial tiene implicancias sobre la liquidez y la circulación de la moneda en un nivel macro, afectando de esta manera el consumo. Las suposiciones en torno a la imposibilidad de ahorrar en dinero de los sectores con bajos recursos deben estudiarse a la luz de experiencias que den cuenta de las condiciones que modelan el ahorro. En la Argentina, la Caja Nacional de Ahorro Postal fue un ejemplo exitoso de ahorro en los sectores populares. La ausencia de condicionamientos para el ingreso al sistema, el valor mínimo de $\$ 1$ a partir del cual se podía realizar un depósito, la cobertura geográfica de la institución a lo largo de todo el país, fueron algunas de las características que llevaron a que en un breve lapso, luego de su creación en 1914, recibiera una masiva adhesión por parte de la población trabajadora. Asimismo, pueden observarse prácticas cotidianas de ahorro no monetario, tales como el aprovisionamiento de alimentos u objetos para su posterior venta (cobre, plásticos, etc.), lo cual indica que no se trata de una cuestión "cultural" - término que frecuentemente se utiliza desde el sentido común para hacer referencia a una supuesta cualidad ontológica (y por esto mismo, ahistórica) de las poblaciones -, sino de las condiciones en las que el ahorro monetario se lleva a cabo.

No obstante, el ejemplo de la Caja Nacional de Ahorro Postal no es traído para argumentar que toda la cuestión se reduciría a la ausencia de instituciones que fomenten el ahorro monetario, ni para concluir que sólo haría falta la implementación de determinados mecanismos para que, sin más, los sectores populares pudiesen incrementar sus ahorros, puesto que no debemos olvidar, en primer lugar, la distancia existente entre las condiciones sociales y económicas de aquel momento y las imperantes hoy en día, la cual hace tropezar cualquier intento comparativo que no muestre recaudos. Y que, no menos importante, las representaciones sociales de dicho sectores en torno a las instituciones bancarias, las ubican en muchos casos especialmente los que nunca han tenido una experiencia con ellas - fuera de toda consideración por ser percibidas como entidades totalmente alejadas de la cotidianeidad de sus vidas, y a las que ellos no podrían ni sabrían cómo acceder. Sin embargo, esta referencia a un ejemplo histórico nos permite desontologizar las disposiciones económicas de los agentes, mostrando que éstas son producto de las condiciones históricas particulares en las que se modelan (Bourdieu, 2008) y que, como ya han visto desde los sectores finan- 
$\operatorname{cieros}^{10}$, no es arbitrario suponer que las prácticas de ahorro y de crédito puedan ser impulsadas con las mediaciones apropiadas, aunque sin dejar de lado la discusión en torno a los fines perseguidos por dicha captación.

Ignacio: Un amigo junta metales toda la semana. Siempre metales. Hace \$250, \$220 los sábados. Trabaja con el padrastro. Hoy por ejemplo vendió poco, hizo $\$ 100$. Hace plata, se compra ropa.

Pablo: ¿Y siempre vende todo o a veces guarda un poco?

I: A veces guarda un poco. Piensa lo que va a gastar en el baile. Si no, si vende todo, él me dijo que después si no lo gasta en cualquier cosa.

Conversando con una residente del asentamiento (Adriana) acerca de un crédito que tomaron ella y su marido para construir una pieza y ampliar su casa, le preguntamos si no habían pensado en ahorrar dinero unos meses y luego llevar a cabo la construcción.

Adriana: Sí. Las otras dos piezas las hicimos así. Comprábamos el material e íbamos construyendo.

Pablo: ¿Y ahorrar en plata?

A: ¿Tener plata en mi casa? Imposible.

P: ¿Te la gastás?

A: Sí, me la gasto yo o mi hija.

P: ¿Cuenta bancaria tienen?

A: No. Ahora estaba pensando en sacar una cuando termine con el crédito. Porque plata en mi casa no puedo tener.

Esto nos indicaría, en primer lugar, que el ahorro se hace bajo las modalidades que tienen a disposición para realizarlo. En este caso, la representación inmediata fue la de comprar el material para ir construyendo, que es otra manera de atesorar - en forma no monetaria - para evitar que la liquidez del dinero devenga un incentivo para el gasto. En segundo lugar, y como corolario, observamos que los dispositivos que reglamentan dichas prácticas tienen efectos concretos que modelan a las mismas y que deben ser tenidos en cuenta al estudiar las prácticas de consumo. Por otra parte, las reglamentaciones bancarias actuales ejercen un claro efecto diferenciador sobre la población, puesto que si las mismas son iguales para todo sujeto de derecho, las condiciones que posibilitan el cumplimiento de dichas normas son

10 En este sentido, una nota del diario El Cronista Comercial titula: "Microahorro: los bancos no tienen políticas para captar el dinero de los más pobres" (11/9/2007). En la misma se lamenta que la Argentina no tenga políticas que vayan en dicho sentido, puesto que las experiencias latinoamericanas mostrarían que el $70 \%$ (sic) de los préstamos, provienen de los ahorros de los "carenciados". 
del todo disímiles según el sector social de que se trate, excluyendo a aquellos que no pueden cumplir con los parámetros de "confiabilidad" requeridos para ser aceptado como cliente en un banco. En otras palabras, lo que opera son normas a partir de las cuales a algunas personas se les otorga "confianza" - es decir, se les da crédito - y a otras no, vale decir, no son confiables, término que evidentemente va más allá de lo meramente económico para designar en términos morales a las personas ${ }^{11}$. Para estos últimos quedan circuitos más heterogéneos de acceso a créditos, habitualmente mediante algún garante o bajo condiciones muy desfavorables en relación a las tasas de interés y a las condiciones de cancelación que ofrecen las entidades bancarias a sectores con suficiente capital económico y simbólico como para resultar "fiables". En nuestro caso, se tratan mayoritariamente de créditos personales otorgados directamente por negocios de venta, los cuales implican no sólo la posibilidad de acceder a bienes de cierta carestía, sino que además ofrecen para el comprador la posibilidad de ser alguien "confiable" a los ojos de vecinos y amigos.

El sistema de crédito que dan algunos negocios de venta de productos, o directamente dedicados a otorgar préstamos inmediatos (tales como "Garbarino", "Efectivo Sí" o "Credilogros"), con intereses que pueden llegar hasta un 57\% anual, se presenta como la única alternativa para muchas personas, permitiendo la adquisición de bienes que de otra forma serían inaccesibles. Con un DNI y un recibo de sueldo o la constancia de percibir un plan social, o mediante una persona que oficie de garante - especialmente cuando no puede comprobar el domicilio propio - pueden acceder a una variedad de bienes que, allende de ser costosos, se tornan mucho más onerosos por las tasas de interés que exigen las casas de venta.

Si tenés la plata te sale mucho más barato que pagar... o sea, te saca del apuro, que vos decís, bueno, doscientos pesos por mes, pero cuando vos terminás de pagar las doce cuotas, porque casi siempre son doce cuotas, terminaste pagando un vagón de guita que por ahí en vez de sacar una tele hubieses sacado dos (Adriana, 22 de Agosto).

A diferencia de otros sectores, donde a partir de la bancarización se "premia" el consumo a crédito -a través de tasas de interés nulas hasta cierta cantidad de cuotas, el descuento de un porcentaje del Impuesto al Valor Agregado

${ }^{11}$ Una nota publicada en el diario El Cronista Comercial el día 30 de agosto de 2006 lleva el título "Oportunidades de crédito para los pobres honrados". Allí puede leerse la afirmación realizada desde una casa de venta de electrodomésticos brasilera, según la cual "los pobres son buenos pagadores, siempre que se los evalúe correctamente". 
(Decreto 1387/2001, art. 48) o de un porcentaje sobre el precio final ${ }^{12}-$, la precariedad laboral y la informalidad es "castigada" mediante recargos, tasas de interés muy superiores a la de los bancos o, en los casos extremos, directamente la exclusión en el acceso a ciertos bienes. Esto provoca que, al margen de lo que representen determinados tipo de gastos en su monto final sobre el total de una economía doméstica, los mismos terminan siendo más onerosos en los sectores de bajos recursos que se hallan excluidos del sistema de financiación bancaria. Pero no se trata sólo de la posibilidad de comprar en cuotas o de los intereses que implican las mismas, sino que esto mismo se extiende sobre un conjunto de bienes y servicios que no requieren a priori la necesidad de un parámetro de "confiabilidad". Casos paradigmáticos son la garrafa de gas y las tarjetas de recarga de telefonía celular, en contraposición al gas natural y a los planes fijos de pago para los teléfonos, de mayor rendimiento y menor costo. En términos generales, se observa que para los pobres vivir implica una mayor cantidad de dinero en términos nominales, y no sólo debido a la menor cantidad de recursos con los que cuentan, sino además a un régimen de consumo que, en los hechos, obtiene mayores ganancias tanto por las reglas que impone como por el ingente mercado al que se dirige.

\section{Endeudamiento y futuro}

Como adelantamos, en las condiciones sociales en las que se insertan las prácticas de consumo dentro del asentamiento el porvenir no es un problema en tanto es problemático el presente. La infinidad de acontecimientos, imprevistos, necesidades, imposibilidades que se presentan tornan al futuro como algo que no es necesario tener como objetivo, dado que la inestabilidad conduce a una imprevisibilidad continua que se contrapone a la esperanza de progreso, subyugando toda planificación futura. De esta manera, el cálculo en el consumo se orienta a la satisfacción de lo cotidiano, puesto que si el campo de lo posible es tan acotado como para esperar una reproducción casi calcada del presente, el "gusto hay que dárselo hoy" y, con suerte, mañana se repita. Sin tener certezas en torno al porvenir, el mismo queda librado a la esperanza, ya sea que esta se deposite en la suerte, en que alguien pueda darles una mano, o en el más difuso - pero no por eso menos fundado en el saber práctico que surge de la experiencia - "algo saldrá".

De esta manera, la sujeción al corto plazo y las incertidumbres sobre el porvenir no ejercen un poder disuasivo sobre el acto de consumo a

\footnotetext{
12 Tal es el caso de los descuentos (que pueden variar entre un 10 y hasta un $40 \%$ ) que se realizan pagando con tarjeta de débito y/o crédito en cadenas de supermercados y comercios de diversos rubros determinados días de la semana.
} 
crédito. Más bien requiere de un cálculo que no especule tanto en función de las posibilidades, sino de las necesidades y/o gustos que de cualquier manera les estarán vedados a futuro. El momento de adquirir siempre trata de corresponderse con el presente, puesto que el porvenir esperable no es sustancialmente diferente al hoy. Teniendo al presente antes que al futuro como campo de visualización, y sin alternativas que posibiliten otras formas de acceso a los bienes, la incertidumbre que genera una compra a crédito queda subsumida en el cálculo por la inmediatez del día a día.

Pablo: ¿Alguna vez tuviste miedo de no poder pagar las cuotas, cuando ibas a comprar algo a Garbarino?

Adriana: El que siempre tuvo eso es mi marido. Él siempre estuvo con que "mirá si nos pasa algo, mirá si les pasa algo a los chicos y no podemos pagar", y yo fui siempre la caradura de "metete igual que no nos va a pasar nada", porque era la única forma de que yo, o los chicos, puedan tener sus cosas...

Excluidos de los sistemas formales de crédito y ahorro, la única posibilidad de conseguir determinados bienes es el ahorro a través de la deuda, es decir, la inversión en el tiempo de la lógica del atesoramiento, comprando a crédito lo que no se tiene posibilidad de ahorrar. En principio no es diferente a lo que sucede en otros sectores sociales, donde la tarjeta de crédito soluciona rápidamente el acceso inmediato. Sin embargo, en nuestro caso, el endeudamiento no se presenta como una opción de compra sino como la única posibilidad concreta de adquirir ciertos bienes, posibilidad que implica quedar sujeto a las condiciones desiguales que conllevan dichos créditos por la exclusión que opera en el mercado formal de dinero.

(...) y ropa, mirá, yo tengo una señora que me da a crédito, porque es la mejor forma de pagarla... está bien, que me cobra, también te digo que me cobra el triple de lo que realmente sale, ¿no?, pero es la única forma de que le puedo comprar y les puedo comprar a todos (Norma, 22 de Agosto).

La mayoría de los jóvenes que pudimos entrevistar dentro del asentamiento no tienen empleo o lo tienen intermitentemente. Algunos colaboran con sus padres cuando estos consiguen un trabajo. Casi todos fueron por algún período "quemeros"13, o lo continúan siendo. El "rebusque" se presenta no como una alternativa, sino como el medio por antonomasia por el cual logran conseguir algo de dinero, el cual se vuelve algo que "va y viene" en su sentido más

13 "Quemeros" es la categoría nativa que designa a las personas que concurren al relleno sanitario del CEAMSE ("la quema") en búsqueda de diversos objetos, ya sea para su utilización o consumo como para su comercialización. 
literal. Como vimos, la forma de percibir el ingreso, la regularidad del mismo, organiza la disposición del dinero y su cálculo en el tiempo. En este sentido, los ingresos que puedan obtenerse tienen una finalidad establecida de antemano ya sea para el plato de comida, para "los vicios" o para pagar alguna cuota -, lo cual les otorga una circulación mucho más rápida. Existe una experiencia concreta de esta circulación, puesto que el dinero no dura, sino que más bien huye.

Yo no puedo ahorrar. Yo tengo plata... porque vos sabés que tenés plata, entonces por ahí ves algo, por ejemplo ahora en el frío, decís "uh, tengo las manos heladas, me voy a comprar un par de guantes", comprás un par de guantes. Decís, tengo frío, uh, me hace falta, qué sé yo, un suéter, una bufanda... vas te comprás eso. Entonces decís "uh, me tomo una sopa o algo así", y vos agarrás, te tomás la sopa y cuando te querés acordar no tenés más nada (Leandro, 22 de Agosto).

Este saber práctico de que la plata "se va", no dura,-sumado al prejuicio fuertemente arraigado de que los "pobres no ahorran"- conduce a una reafirmación de que efectivamente ellos "no pueden ahorrar". Por un lado nos hallamos frente a las modalidades que regulan el ahorro; y por el otro, con la vivencia concreta de que los ingresos siempre se hallan muy por debajo de las necesidades y/o aspiraciones.

Sin embargo, esto encierra la paradoja de que por el mismo movimiento en el que el dinero "huye", es decir, se va, se gasta, también viene, vuelve de alguna manera. Por este motivo muchas veces se observa liviandad en su uso, puesto que sin estar atados a las pautas y obligaciones morales de previsión de otros sectores sociales ${ }^{14}$, los jóvenes entrevistados utilizan el dinero para vivir hoy, por lo que las expectativas giran en torno al presente y no en cómo ahorrar para mañana, atados a la experiencia individual - y colectiva - de que el dinero nunca sobra ni alcanza y de que día a día se repetirá, de alguna manera, la misma escena. De esta manera, el presente debe ser aprovechado,

${ }^{14}$ Siguiendo a Bataille, podemos decir que la pregunta moral por excelencia sería “¿qué tengo que hacer (qué debo hacer o qué tengo interés en hacer o qué tengo ganas de hacer) aquí (en este mundo en que tengo mi naturaleza humana y personal) y ahora?" (Bataille, 1972, p. 205). En este sentido, las previsiones en pos de asegurar continuamente el futuro, esa forma de cálculo que trata de conjurar la muerte, el miedo que ella provoca y que se encuentra al final de todos los temores sobre el porvenir, sería una manera contraria al vivir hoy que pudimos observar en nuestro caso de estudio. Y sin embargo, paralela y contradictoriamente, las mismas clases medias son especialmente proclives a padecer los infortunios de unas aspiraciones que no se corresponden con sus posibilidades objetivas, por lo que se ven obligadas a vivir a crédito para obtener las últimas modas, coaccionadas por la lucha simbólica que las lleva a querer distinguirse de las capas más bajas de la sociedad y asemejarse a las más altas (Bourdieu, 1999). 
especialmente de manera colectiva, es decir, en solidaridad con los otros, pasar un "bueno momento", "darse el gusto", que no es más que una forma de reafirmar los lazos sociales que actúan como el único capital que poseen frente al porvenir ${ }^{15}$.

El siguiente es un extracto de conversación registrada con algunos jóvenes del barrio, entre 16 y 26 años, todos amigos entre sí. En un momento de la misma, imaginamos que cada uno podía disponer de $\$ 500$ en efectivo para utilizarlos como quisieran:

Pablo: Ustedes ¿hacen empeño cuando están ajustados?

Damián: O sea, no empeño, pero por ejemplo, si tenemos aluminio, o lo que más sale...

Adrián: Si tenés electrónicos y estás ajustado de plata, lo vendés...

D: Bueno, pero yo no tengo nada electrónico, yo te digo de mi situación...

Leandro: O por ahí, vos tenés la suerte, y haciendo cualquier otra cosa traés un aparato o algo y querés plata y lo vendés. O por ahí alguna ropa tuya que no te hace falta o no la usás nunca y a uno le gusta, y agarrás y se la vendés. Es muy informal acá, a veces nosotros nos la rebuscamos para tener un poco de plata.

D: Es como una forma de sobrevivir para nuestra forma.

L: La forma así de nosotros, para tener plata...

R: Para movernos, por ejemplo si queremos comprarnos un pucho, o una gaseosa...

L: Mi viejo siempre me dice: "hacé algo, aunque sea una changuita, algo para mantenerte los vicios".

R: Sí, más cuando estás de novio, es más el gasto.

L: Qué vas a estar, ¿pidiéndome plata para agarrar y llevar a tu novia a tomar un helado? O por ahí tenés un par de billetes, ponele un quinientos como decís vos, y te vas a comer a un lugar bien piola con la mina.

R: Es como la forma que tiene cada uno de expresar el amor que tiene por la persona que la quiero, o con quien está. Pero si yo tuviera lo 500 mangos, sí, haría como dice L. Iría a un lugar piola piola y comeríamos ahí.

P: Ahora, si sabés que después de esos quinientos mangos no comés en todo el mes... o bueno, seguís como estabas antes...

${ }^{15}$ En este sentido, “(...) la presencia en el presente que se afirma en la preocupación por aprovechar los buenos momentos y por tomar los tiempos conforme vienen es, en sí, una afirmación de solidaridad con los otros (que son, por lo demás, la única garantía presente frente a las amenazas del porvenir), en la medida en que esta especie de inmanentismo temporal es un reconocimiento de los límites que definen la condición" (Bourdieu, 1999, p. 181). 
L: Bueno, pero si la guacha te quiere, que te invite a comer ella... (risas) Hoy por vos, mañana por mí.

R: Si yo sé que tengo para tirar, para mí, me daría ese gusto.

P: Cuando pueden, ¿se dan gustos?

L: Los gustos te los das, siempre.

$\mathrm{R}$ : O vas, o le comprás algo, un regalo, una remera o lo que sea, ¿entendés? Tampoco te sale tan caro.

L: Hay muchas formas de gastar los quinientos. Por ejemplo, vos estando solo, los vas a gastar para vos, para mantenerte vos, para gastar en cosas que vos quieras. En cambio, si estás con alguien, si estás en pareja o estás viviendo con tus viejos, vas a colaborar con tus viejos, vas a gastar también con tu chica, o algo, y después vas a querer gastar en vos y cuando te das cuenta no tenés nada. ¿O no? Nosotros cada vez que tenemos plata decimos "ah, tengo plata..." gastas, gastas y cuando te querés dar cuenta tenés dos pesos en el bolsillo.

Entrevistador: ¿Prefieren pasar el momento ahora y después verán...?

L: Y sí, es como decías vos, ¿viste que nos damos el gusto con la comida? Bueno, nosotros nos damos el gusto con salir, gastar, divertirnos un rato y después... bueno, vemos si se nos da el gusto de vuelta.

Sin poder ubicarse en torno a una actividad concreta, la misma forma de vida que obliga a recurrir a las más variadas actividades otorga la confianza de que algo podrá hacerse, incluso vender lo que hoy se tenga la posibilidad de comprar. En este sentido, como vimos, en las prácticas de consumo actúan otras tantas formas de cálculo que no se relacionan con la contabilidad de recursos, y que sin embargo actúan al momento de realizar un gasto. Las operaciones mentales que se producen no son más que otras tantas operaciones colectivas -vinculadas tanto a las condiciones de un medio social dado cuanto a las relaciones simbólicas que en el se desarrollen-, las cuales son individuadas en prácticas concretas que reproducen el mundo de lo esperable. El cálculo no es sólo un procedimiento lógico-matemático, sino que además intervienen en él experiencias previas, esperanzas, expresiones simbólicas y hábitos que llevan a que el gasto no sea en cualquier caso una mera optimización de los recursos, sino el producto de un complejo entramado de operaciones en las que la escasez ocupa sólo una parte. Así, al momento de consumir interviene, por caso, la certeza de que podrá recurrirse al empeño del objeto adquirido, la esperanza de conseguir una "changa", o incluso la "obligación" de llevar a la persona querida a un lugar "piola", despreocupándose por el porvenir y ateniéndose a la satisfacción de lo inmediatamente asequible. 
Los pequeños ahorros que puedan lograrse se realizan con un destino concreto, algún artículo de un valor lo suficientemente elevado como para que no puedan comprarlo al contado, pero que no obstante se ubica dentro del campo de los posibles a los cuales "pueden" acceder. Puesto que este "pueden" es construido socialmente más que en función del dinero disponible, lo que supone operaciones mucho más complejas que lo que podría ser un determinismo económico sobre los hábitos de consumo. Como expresa Bourdieu,

Sólo en la experiencia imaginaria (la del cuento, por ejemplo), que neutraliza el sentido de las realidades sociales, el mundo social reviste la forma de un universo de posibles igualmente posibles para todo sujeto posible. Los agentes se determinan con relación a índices concretos de lo accesible y de lo inaccesible, del "es para nosotros" y del "no es para nosotros", división tan fundamental y tan fundamentalmente reconocida como la que separa lo sagrado y lo profano (Bourdieu, 2007, p. 104).

Pensar las prácticas económicas - como cualquier tipo de prácticas requiere de un análisis de procesos y dispositivos por los cuales se construye y sostiene una normalización de dichas prácticas. Con esto queremos señalar que la modalidad en que se lleva a cabo el consumo, el crédito y el ahorro, y las relaciones que se establecen a partir de estas cuestiones, no son fortuitas ni pueden ser estudiadas como el mero resultado de una consideración lógica sobre la utilización óptima de recursos. Antes bien, se encuadran en una serie de condicionamientos que hacen a una determinada gubernamentalidad (Foucault, 2007a; 2007b), es decir, a una manera de gobernar la conducta de los hombres tendiente a crear las condiciones de posibilidad para que una institución como el libre mercado pueda funcionar (Grenier y Orléan, 2007), normalizando los actos a través de un entramado jurídico e institucional que facilite el desenvolvimiento pretendidamente natural de los "fenómenos económicos". En tanto las relaciones sociales no se inscriben en un vacío institucional, el derecho actúa positivamente en función de construir la "naturalidad" de la dimensión económica de las relaciones, de asegurar el buen desempeño de las mismas (Landau, 2008).

Sin embargo, esto no significa que dichos mecanismos actúen sobre una tabula rasa, sino que al extenderse sobre el conjunto de la población se inscriben en contextos sociales específicos con lógicas heterogénas y particulares, sobre los cuales generan efectos diferenciados y diferenciadores. En efecto, los requisitos para el acceso al crédito, a una cuenta bancaria, las tasas de interés, los impuestos, son algunas de estas maneras de gobernar a las personas, en tanto delimitan los mecanismos formales - al mismo tiempo 
que dejan espacio para los informales-por los que se disponen las prácticas de los agentes. Pero, como vimos, las implicancias serán disímiles de acuerdo a los universos sociales en cuestión, puesto que las desiguales condiciones materiales (pero también simbólicas) conllevan formas diferenciadas de prácticas que, aunque igualmente económicas, pueden ser aprehendidas como económicamente heterogéneas. En este sentido, quisimos dar cuenta de cómo actúan concretamente algunos de estos dispositivos y mostrar los efectos singulares que tienen en la conformación de determinados usos en un sector con bajos ingresos, en virtud tanto de los desiguales accesos que posibilitan e imposibilitan el gasto y el ahorro como de las visiones que sobre el porvenir se forjen.

\section{Referencias}

BATAILLE, Georges. La experiencia interior. Madrid: Taurus, 1972.

BOURDIEU, Pierre. La distinción. Criterio y bases sociales del gusto. Madrid: Taurus, 1999.

BOURDIEU, Pierre El sentido práctico. Buenos Aires: Siglo XXI Editores, 2007.

BOURDIEU, Pierre. La fabricación del habitus económico. Crítica en Desarrollo, Buenos Aires, n. 2, p. 15-42, 2008.

DUFY, Caroline; WEBER, Florence. Más allá de la Gran División: sociología, economía y etnografía. Buenos Aires: Antropofagia, 2009.

FIGUEIRO, Pablo: El gasto improductivo en los sectores subalternos: aproximaciones a las lógicas sociales del consumo en un asentamiento del Partido de General San Martín. Tesis de Maestría. Instituto de Altos Estudios Sociales de la Universidad Nacional de San Martín, Buenos Aires, febrero de 2009.

FOUCAULT, Michel. El nacimiento de la biopolitica. Buenos Aires: Fondo de Cultura Económica, 2007a.

FOUCAULT, Michel. Seguridad, territorio y población. Buenos Aires: Fondo de Cultura Económica, 2007b.

FOUCAULT, Michel. La verdad y las formas jurídicas. Buenos Aires: Gedisa, 2007c.

GRENIER, Jean Yves; ORLÉAN, André. Michel Foucault, l'économie politique et le libéralisme. Annales, Francia, v. 62, n. 5, p. 1155-1182, 2007.

LANDAU, Martín. Gobernar por la economía: apuntes foucaultianos para pensar la relación entre ciencia y derecho. Crítica en Desarrollo, Buenos Aires, n. 2, p. 217-224, 2008.

MILLER, Daniel: Ir de compras: una teoría. México: Siglo XXI Editores, 2000.

REYGADAS, Luis. La apropiación: destejiendo las redes de la desigualdad. México: Anthropos Editorial, 2008.

ZELIZER, Viviana. The social meaning of Money. Princeton: Princeton University Press, 1997. 
ZELIZER, Viviana. Circuits within capitalism. In: NEE, V.; SWEDBERG, R. (Ed.) The economic sociology of capitalism. Princeton: Princeton University Press, 2005a. p. 289-322.

ZELIZER, Viviana. Culture and consumption. In SMELSER, N.; SWEDBERG, R. (Ed.). Handbook of economic sociology. Princeton: Princeton University Press/New York: Russell Sage Foundation, 2005b.

\section{Fuentes}

Comunicación “A” No 2590 del Banco Central de la República Argentina del 19 de septiembre de 1997. Disponible desde Internet en: <http://www.bcra.gov.ar/>.

Diario El Cronista Comercial del 30/08/2006 y del 11/09/2007. 